\title{
Production of Superoxide Dismutase by Deinococcus radiophilus
}

\author{
Young Sun Yun and Young Nam Lee* \\ Division of Life Sciences and Research Institute for Biotechnology, Chungbuk National University, \\ Cheongju, Chungbuk 361-763, Korea
}

Received 20 November 2002, Accepted 26 December 2002

The production of superoxide dismutase (SOD) varied in Deinococcus radiophilus, the UV resistant bacterium, depending upon different phases of growth, UV irradiation, and superoxide treatment. A gradual increase in total SOD activity occurred up to the stationary phases. The electrophoretic resolution of the SOD in cell extracts of $D$. radiophilus at each growth phase revealed the occurrence of MnSOD throughout the growth phases. The SOD profiles of $D$. radiophilus at the exponential phase received oxidative stress by the potassium superoxide treatment or UV irradiation also revealed the occurrence of a single SOD. However, these treatments caused an increase in SOD activity. The data strongly suggest that $D$. radiophilus has only one species of SOD as a constitutive enzyme, which seems to be a membrane-associated protein.

Keywords: Constitutive enzyme, Deinococcus radiophilus, Membrane-associated SOD, Oxidative stress, Superoxide dismutase, UV resistance

\section{Introduction}

Superoxide dismutases (SODs, EC1.15.1.1) protect cells from the toxicity of superoxide radicals $\left(\mathrm{O}^{-}\right)$which are generated in cells by various causes, such as aerobic respiration, UV, ionizing radiations (X-ray, $\gamma$-ray, cosmic ray), cigarette smoking, and some redox-cycling drugs (Fridovich, 1995; Halliwell and Gutteridge, 1998; Cho and Song, 2000; Storz and Zheng, 2000). All SODs are metalloenzymes such as MnSOD, FeSOD, and Cu/Zn SOD. Also, an occurrence of NiSOD was reported in the Streptomyces species (Youn et al., 1996). Metal ions at the active site of SODs are different, depending upon cell types; $\mathrm{Cu} / \mathrm{ZnSOD}$ is found exclusively in cytosols of eukaryotes and rarely in prokaryotes. In contrast,

*To whom correspondence should be addressed.

Tel: 82-43-261-2301; Fax: 82-43-264-9600

E-mail: ynlee@ cbucc.chungbuk.ac.kr
MnSOD is present in both the prokaryotes and mitochondria of eukaryotes; FeSOD is found in the cytosol of prokaryotes, as well as in the chloroplasts of some green plants (Halliwell and Gutteridge, 1998).

The most peculiar feature of the Deinococcus species is their extreme resistance against UV and ionizing radiations and oxidative stress (Evans and Moseley, 1983; Murray, 1986; Mueller et al., 1996; Battista, 1997). Although it could be speculated that the unusual radio-resistance is attributed to their morphological characteristics (including a thick cell wall of several distinct layers and membrane-bound carotenoid pigment), one could easily assume that the operation of the efficient scavenging systems against the reactive oxygen species (ROS) and the repairing potential of the damaged cellular components that are mediated by toxic oxidants are highly engaged in the extreme radio-resistance of Deinococcus. Extensive studies on UV resistance in regard to the repairing genes for damaged DNA (Evans and Moseley, 1983; Gutman et al., 1993; Daly et al., 1994; Agostini et al., 1996; Narumi et al., 1997; Bauche and Laval, 1999; Kim et $a l ., 2002$ ) and the entire genome analysis of D. radiodurans that was reported in recent years (White et al., 1999; Makarova et al., 2001) would be insufficient to understand the radio-resistant nature of Deinococcus. Therefore, attention has been paid to the scavenging enzymes against the reactive oxygens in relation to the UV resistance. Also, studies on hydroperoxidases (catalases) that eliminate peroxide radicals were conducted in $D$. radiophilus that possess three isotypes of catalases (Lee and Lee, 1995; Oh and Lee, 1998; Yun and Lee, 2000). Nevertheless, other ROS-scavenging enzymes in Deinococcus have not yet been investigated. Since SODs, along with catalases, play key roles in protecting cells against toxic oxidants, the production of SOD in Deinococcus radiophlius was investigated.

\section{Materials and Methods}

Bacterial culture and chemicals Deinococcus radiophilus ATCC 27603 was cultured in a TYGM medium (1\% tryptone, 
$0.5 \%$ yeast extract, $0.2 \%$ glucose, and $0.2 \%$ L-methionine) at $30^{\circ} \mathrm{C}$ with continuous aeration at $150 \mathrm{rpm}$ (Soung and Lee, 2000; Yun and Lee, 2001). Pseudomonas aeruginosa was grown in a LuriaBertani (LB) medium. Bacterial growth was recorded with an optical density at $600 \mathrm{~nm}\left(\mathrm{OD}_{600}\right)$ (DU-65 Spectrophotometer, Beckman). Most of the medium constituents and chemicals including riboflavin, nitro blue tetrazolium (NBT), tetramethylethylenediamine (TEMED), KCN, pyrogallol, cacodylic acid, diethylenediamine-pentaacetic acid (EDPA) potassium superoxide, and materials for polyacrylamide gel electrophoresis were purchased from the Difco Lab. (Detroit, USA) and Sigma Chemical Co. (St. Louis, USA), respectively.

SOD assay and protein quantification The SOD activity in the cell-free extracts that were prepared by ultrasonic disruption was assayed by the method of pyrogallol autooxidation. The pyrogallol autooxidation by superoxide radicals, which is generated chemically from cacodylic acid and EDPA, was measured in the presence of the SOD enzyme. One unit of SOD was defined as the amount of enzyme that causes 50\% inhibition of the pyrogallol autooxidation rate at $420 \mathrm{~nm}$ (Marklund and Marklund, 1974). The protein concentration was determined by the method of Lowry et al. (1951).

Gel electrophoresis Proteins in the cell-free extract that was prepared by ultrasonic disruption (Soung and Lee, 2000; Yun and Lee, 2001) were resolved by nondenaturing polyacrylamide gel electrophoresis (PAGE) in a Tris-glycine buffer (Gersten, 1996).

Activity staining of SOD Visualization of the SOD bands that were resolved on a polyacrylamide gel was made by a method (Beauchamp and Fridovich, 1971) modified by Chou and Tan (1990). The gels were soaked in $490 \mathrm{uM}$ NBT for $20 \mathrm{~min}$, then in a solution that contained $28 \mathrm{mM}$ TEMED, $28 \mathrm{uM}$ riboflavin, and $36 \mathrm{mM}$ potassium-phosphate buffer $(\mathrm{pH} 7.8)$ for $15 \mathrm{~min}$. Then, the gels were illuminated with a fluorescent lamp for 5-15 min to visualize white bands of SOD activity in the blue background. Density of the SOD bands on the gel was measured by densitometry with either gel Doc 1000 (Molecular analyst software, version 1.4.1) from Bio-RAD (Hercules, USA), or Kodak electrophoresis documentation and analysis system (KODAK 1D Image Analysis, Tokyo, Japan).

Distinction between the FeSOD and MnSOD bands was made by soaking the gels for $60 \mathrm{~min}$ at room temperature in a solution of $20 \mathrm{mM} \mathrm{H}_{2} \mathrm{O}_{2}$ and $50 \mathrm{mM}$ potassium phosphate $(\mathrm{pH} \mathrm{7.8)}$ before activity staining for SOD. This treatment caused the selective inactivation of FeSOD (Chou and Tan, 1990).

SOD induction by either superoxide treatment or UV irradiation The survival of $D$. radiophilus upon exposure to potassium superoxide and UV irradiation was studied prior to an induction study of SOD by superoxide treatment and UV irradiation. Exponentially-grown $D$. radiophilus was subcultured into a new TYGM medium in order to give an initial $\mathrm{OD}_{600}$ of 0.1 . When the culture reached an $\mathrm{OD}_{600}$ of 0.2 , potassium peroxide was added to the culture with final concentrations from 0-100 mM. The culture was then further incubated for $60 \mathrm{~min}$ at $30^{\circ} \mathrm{C}$. Properlydiluted cell cultures with $50 \mathrm{mM}$ potassium phosphate buffer $(\mathrm{pH}$
7.0) were plated on TYGM agar in triplicate for the colony-forming units (CFU). The cells of the culture that reached an $\mathrm{OD}_{600}=0.2$ were harvested by centrifugation. They were washed three times and resuspended in $50 \mathrm{mM}$ potassium phosphate buffer, $\mathrm{pH}$ 7.0. The cell suspension in a Petri dish (without lid) was UV irradiated with a UV lamp (Minuvis, Desaga, Heidelberg, Germany). The UV dose $\left(0-1500 \mathrm{~J} / \mathrm{m}^{2}\right)$ was measured using a VLX-3W radiometer (Vilber Lourmat, Marne La Vallee, France). Ten $\mathrm{ml}$ of the irradiated cell suspension was placed in the test tube, then vortexed and subjected in order to measure CFU. For comparison, a parallel experiment was run with $P$. aeruginosa that was grown in a LB medium. CFUs of $D$. radiophilus and $P$. aeruginosa were recorded at 48 and $24 \mathrm{~h}$ of incubation on the TYGM and LB agar plates, respectively. For an induction study of SOD, the cultures of $D$. radiophilus $\left(\mathrm{OD}_{600}=0.2\right)$ that received different concentrations of potassium superoxide $(0-20 \mathrm{mM})$ were further incubated for $60 \mathrm{~min}$ at $30^{\circ} \mathrm{C}$. Then, the cell-free sonic extract was prepared with the cells that were harvested. The UV-irradiated cells, as previously described, were allowed a $60 \mathrm{~min}$ incubation at $30^{\circ} \mathrm{C}$ before preparation of the cell-free sonic extract.

Cellular localization of SOD The cells that were harvested at the stationary phase were suspended in $30 \mathrm{mM}$ Tris- $\mathrm{HCl}$ buffer $(\mathrm{pH}$ 8.0) that contained $20 \%$ sucrose and $1 \mathrm{mM}$ EDTA at a ratio of $1 \mathrm{~g}$ of wet cells in $80 \mathrm{ml}$ of the buffer (Yun and Lee, 2000). After a $10 \mathrm{~min}$ incubation at $30^{\circ} \mathrm{C}$, the supernatant and cell pellet were fractionated by centrifugation. The proteins on the supernatant (sucrose-shocked fluid) that was dialyzed against $50 \mathrm{mM}$ potassium phosphate buffer ( $\mathrm{pH}$ 7.0) were concentrated to several folds by ultrafiltration (PM-10 membrane, Amicon, Beverly, USA). The cell pellet was followed by a cold-wash step; suspension of the pellet in cold-distilled water allowed a 10 min incubation in an ice bath in order to release the loosely-bound proteins on the pellet surface. The cold wash supernatant and cell pellet were fractionated by centrifugation. Proteins in the sucrose-shocked cell pellet were released by sonic disruption. SOD in each preparation was resolved by PAGE, followed by activity staining. Since catalase- 2 and catalase-3 of D. radiophilus are known as members of membrane and cytosol proteins, respectively (Yun and Lee, 2000), then catalase activity staining of the gel is simultaneously done for the references of fractionation.

\section{Results and Discussion}

SOD is the most indispensable enzyme for protecting the cells from the toxicity of the reactive oxygen species that are generated during aerobic respiration for energy production. The SOD activity of $D$. radiophilus showed a gradual increase along with growth phases, as shown in Fig. 1. The growthdependent increase of SOD activity in aging cells could be attributed to the accumulation of endogenous oxidants during the prolonged respiratory activity, as reported in other organisms, including E. coli, Caulobacter crescentus, Streptomyces coelicolor, Arcobacter nitrofigilis, and Schizosaccharomyces pombe (Farr and Kogoma, 1991; Schnell and Steinman, 1995; Kim et al., 1996; Park and Han, 


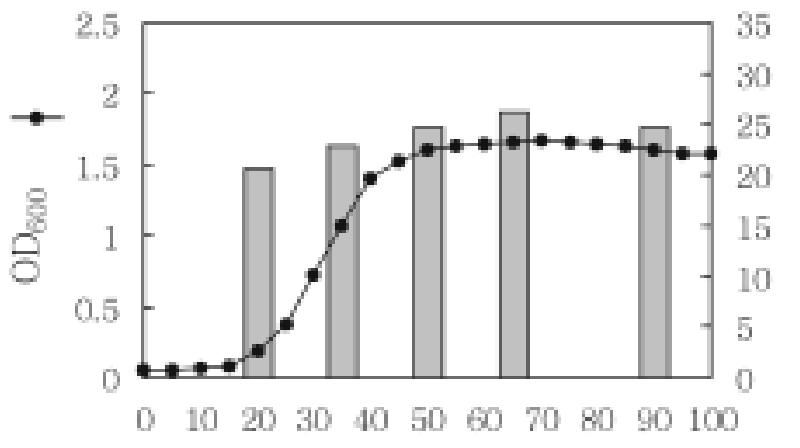

Time (h)

Fig. 1. Growth and SOD activity of D. radiophilus. Exponentially grown $D$. radiophilus in TYGM medium was subcultured into a new TYGM medium to give an initial $\mathrm{OD}_{600}=0.1$. Growth $\left(\mathrm{OD}_{600}\right)$ and SOD activity were determined as described in the material and methods.

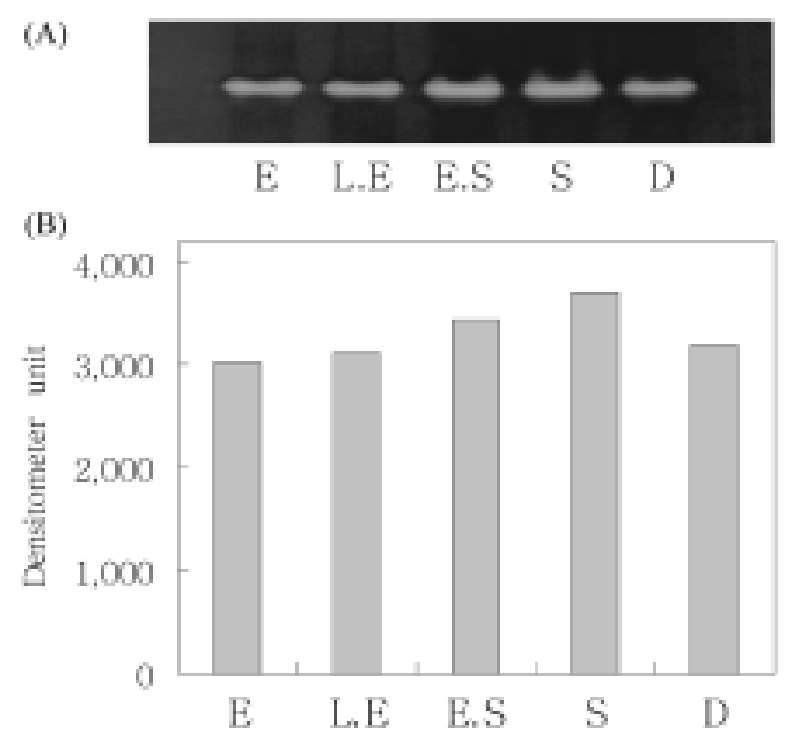

Fig. 2. Production of SOD by $D$. radiophilus during growth phases. (A) Proteins in cell-free extracts were resolved on $10 \%$ gel. Activity staining of SOD band was made by the method described in the text. (B) Densitometry of SOD band. 1. exponential phase $(\mathrm{E}$ at $20 \mathrm{~h})$ 2. late exponential phase (L.E at $35 \mathrm{~h}) 3$. early stationary phase $(\mathrm{E} . \mathrm{S}$ at $50 \mathrm{~h}) 4$. stationary phase $(\mathrm{S}$ at $65 \mathrm{~h}) 5$. death phase (D at $90 \mathrm{~h}$ ). Densitometry was done with gel Doc 1000, Bio-RAD (Molecular analyst software, version 1.4.1).

1997; Cho et al., 2001). The electrophoretic resolution of SOD in the cell-free extracts that were prepared with $D$. radiophilus cultures during each growth phase revealed the occurrence of single SOD (Fig. 2). The SOD band at the stationary phase was about $20 \%$ denser when compared with that at the early exponential phase. The Deinococcus species, the obligate aerobics, are extraordinarily resistant to UV- and ionizing-radiations. For example, $D$. radiodurans can survive exposure up to 30,000 Gy of ionizing radiation (Madigan et
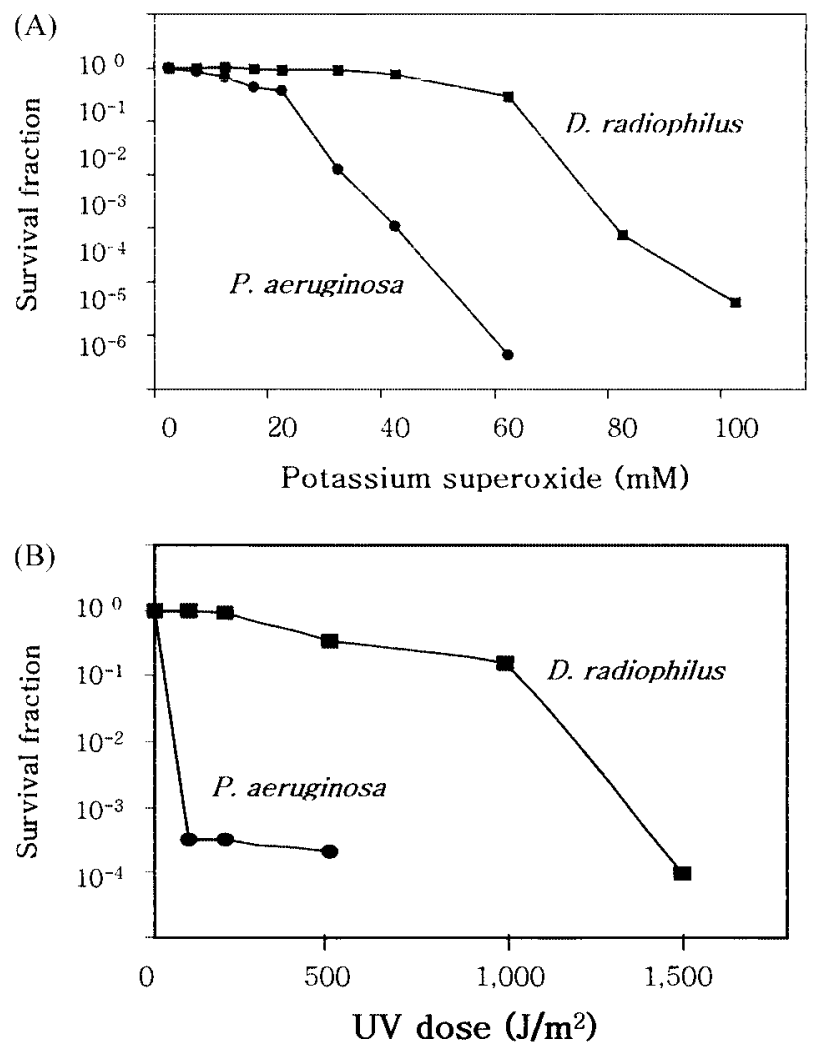

Fig. 3. Survival of $D$. radiophilus exposed to potassium superoxide (A) and UV radiation (B). The bacterial cultures $\left(\mathrm{OD}_{600}=0.2\right)$ either received potassium superoxide or UV irradiated (B) were incubated for $60 \mathrm{~min}$ at $30^{\circ} \mathrm{C}$. The treatedcells were collected, washed and suspended in the sterilized $0.85 \%$ saline solution and plated on TYGM agar. Survival fraction was represented as colony forming units (CFUs) of the treated-culture/CFUs of non-treated culture.

al., 2000). In addition to the deleterious effect of UV radiation on DNA, the UV light attributes various cell damages to generating-reactive oxygen intermediates (Tyrrell, 1991). The high resistance of $D$. radiophilus to exogenous superoxide and UV radiation is depicted in Fig. 3, there is a nearly 50\% survival, even with a $60 \mathrm{mM}$ potassium superoxide treatment (Fig. 3A) and $1,000 \mathrm{~J} / \mathrm{m}^{2}$ of UV dose (Fig. 3B). Treatment of cells with varied concentrations of potassium superoxide caused a differential increase of SOD, that is, ca. $60 \%$ increase of the level of untreated cells at $15 \mathrm{mM} \mathrm{KO}_{2}$ (Fig. 4A). However, an intensity of the SOD band that was obtained from the $15 \mathrm{mM} \mathrm{KO}$ treated-cells was about $20 \%$ denser when compared with that of the non-treated cells. The discrepancy in the increase of SOD between the specific activity in cell-free extract and resolution on the gel could be due to the different systems of assay. A similar result of the SOD production was obtained when the cells were exposed to UV (Fig. 5). All of these data suggest that D. radiophilus SOD is a constitutive enzyme whose activity is inclined to increase with environmental stresses. D. radiophilus SOD was 
(A)

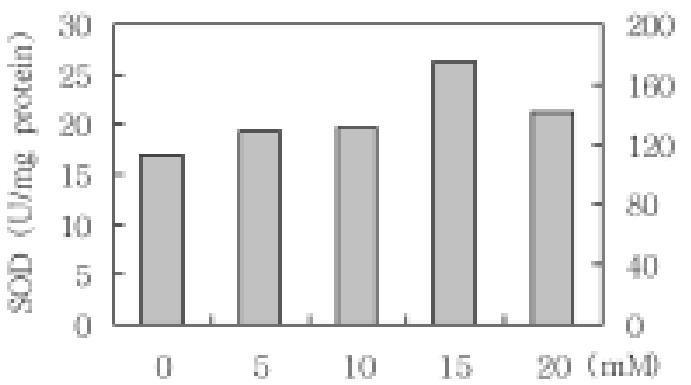

(B)

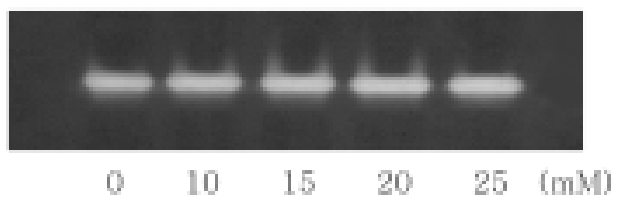

(C)

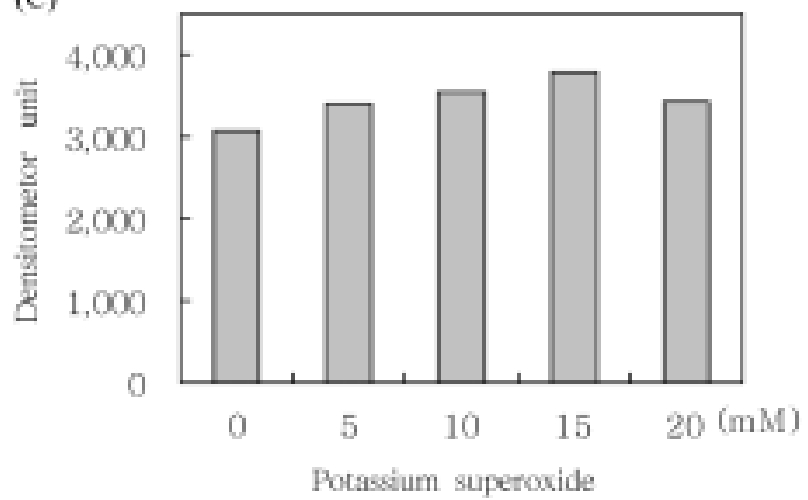

Fig. 4. Production of $\mathrm{SOD}$ by $D$. radiophilus treated with potassium superoxide. (A) Cells in exponential phase were treated with potassium superoxide for $60 \mathrm{~min}$. SOD and protein concentration were determined as described in the text. Increase (\%) of specific activity of SOD in the potassium superoxide treated cells was compared with that of untreated cells. (B) Proteins in cell-free extracts made with the potassium superoxide treated cells were resolved on $10 \%$ gel and followed by activity staining by the method described in the text (Chou and Tan, 1991). (C) Densitometry of SOD bands on gel with gel Doc 1000, Bio-RAD (Molecular analyst software, version 1.4.1).

reported as MnSOD in a previous paper (Yun and Lee, 2001), and it was reconfirmed by sensitivity studies (data not shown). MnSOD is insensitive to both hydrogen peroxide and cyanide. In contrast, CuZnSOD is sensitive to hydrogen peroxide as well as cyanide. FeSOD is sensitive to hydrogen peroxide but not to cyanide (Chou and Tan, 1990).

Reports on the iso-enzymes that show distinct physiological roles with different cellular localizations in prokaryotic cells were made in many instances (Gregory et al., 1973; Goldberg and Hochman, 1989; Kim et al., 1996; St. John and Steinman, 1996; Yun and Lee, 2000; Li et al., 2002). In D. radiophilus, the membrane-associated catalase- 2 is a constitutive enzyme, whereas the cytosolic catalase-3 is an inducible enzyme that responds to environmental stress (Yun and Lee, 2000). Another example of these instances is a recent report on the
(A)

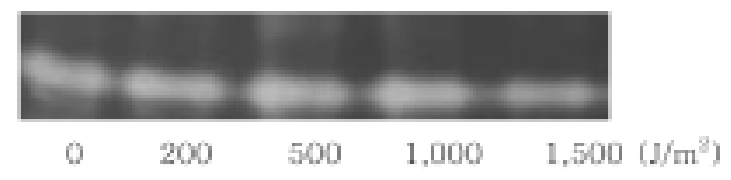

(B)

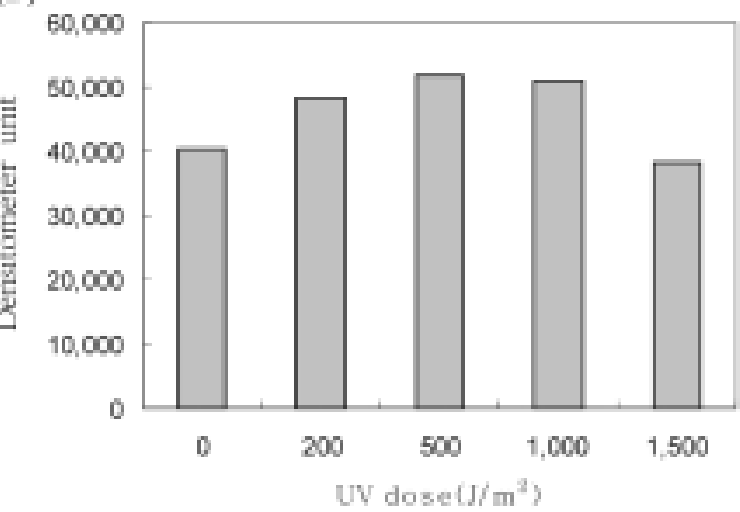

Fig. 5. Production of SOD by D. radiophilus irradiated with UV. (A) Cells of exponential phase were given the UV dose and incubated for $60 \mathrm{~min}$. SOD and protein concentration were determined by the methods described in the text. Proteins in cellfree extracts were resolved on $10 \%$ polyacrylamide gel and SOD bands were visualized by activity staining. (A) Electrophoretic resolution of SOD from the UV treated cells. (B) Densitometry of SOD bands on gel with Kodak Electrophoresis documentation and analysis system (KODAK 1D Image Analysis).

(A)

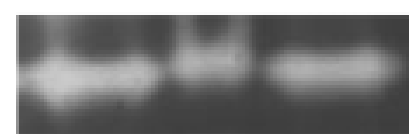

$\begin{array}{lll}1 & 2 & 3\end{array}$

(B)

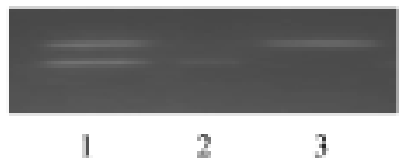

Fig. 6. Cellular localization of SOD. Resolution of $D$. radiophilus SOD in each fraction by PAGE and activity staining. Each well was loaded with $20 \mathrm{ug}$ of protein. (B) Resolution of iso-catalase occurred in $D$. radiophilus by PAGE and catalase activity staining. The details of catalase activity staining were found in the reference (Yun and Lee, 2000). Lane 1, Cell-free extract of whole cell at stationary phase; lane 2, Supernatant of sucrose-shocked cells (= cytosol fraction); lane 3, Sonic supernatant of sucrose-shocked pellet (= membrane fraction).

induction of FeSOD during heterocyst differentiation and localization of MnSOD in the thylakoid membrane in Cyanobacteria Anabaena sp. (Li et al., 2002). Electrophoretic resolution of the protein in the preparations that were obtained from the sucrose-shocked cells of $D$. radiophilus revealed the omni-presence of SOD in both the cytosol and membraneassociated fractions. However, there was an obvious difference in SOD abundance between the two fractions, ca. three-fold denser in the intensity of the SOD band $(145,000$ 
densitometer unit, DU) in the membrane fraction when compared with that of the cytosol fraction (56,000 DU) (Fig. $6)$. However, we consider that $D$. radiophilus SOD is a membrane-associated protein because of its abundance and clear presentation of iso-catalases that are employed as the reference proteins of each fraction. We assume that SOD in cytosol could be attributed to either the not-yet membraneincorporated SOD that occurred in cytosol, or SOD that is released from the membrane into the cytosol fraction during osmotic shock by sucrose. All of our data suggests that $D$. radiophilus possesses a sole MnSOD, which is a constitutive enzyme and responsible for scavenging the superoxide radicals either internally or externally. Therefore, one might easily assume the immense role of this MnSOD to $D$. radiophilus in surviving environmental stresses, including UV irradiation and oxygen stress. Further studies of the regulation of ROS-scavenging enzymes (including SOD, hydroperoxidase, glutathione reductase, and others in Deinococcus species) are required in order to understand its radioresistant physiology.

Acknowledgments This work was supported by grant No. ROS-2001-000-00320-0 from the Basic Research Program of the Korea Science and Engineering Foundation. We thank Ms. J. Soung for the confirmation of SOD localization data by repeated experiments.

\section{References}

Agostini, H. J., Carroll, J. D. and Minton, K. W. (1996) Identification and characterization of $u v r A$, a DNA repair gene of Deinococcus radiodurans. J. Bacteriol. 178, 759-765.

Battista, J. R. (1997) Against all odds: The survival strategies of Deinococcus radiodurans. Ann. Rev. Microbiol. 51, 203-224.

Bauche, C. and Laval, J. (1999) Repair of oxidized bases in the extremely radiation-resistant bacterium Deinococcus radiodurans. J. Bacteriol. 181, 262-269.

Beauchamp, C. and Fridovich, I. (1971) Superoxide dismutase: improved assays and assay applicable to acrylamidegels. Anal. Chem. 44, 276-287.

Cho, Y. and Song, K. B. (2000) Effect of $\gamma$-radiation on the molecular properties of bovine serum albumin and blactoglobulin. J. Biochem. Mol. Biol. 33, 133-137.

Cho, Y. -W., Park, E. -H., Ahn, K. -S., Kim, D. and Lim, C. -J. (2001) Growth-dependent variations in antioxidant and redox enzymes activities of Schizosaccharomyces pombe. J. Biochem. Mol. Biol. 34, 278-283.

Chou, F. I. and Tan, S. T. (1990) Manganese (II) induces cell division and increases in superoxide dismutase and catalase activities in an aging Deinococcal culture. J. Bacteriol. 172, 2029-2035.

Daly, M. J., Ouyang, L., Fuchs, P. and Minton, K. W. (1994) In vivo damage and RecA-dependent repair of plasmid and chromosomal DNA in the radiation-resistant bacterium Deinococcus radiodurans. J. Bacteriol. 176, 3508-3517.

Evans, D. M. and Moseley, B. E. (1983) Roles of the uvs C, $u v s D, u v s E$ and $m t c A$ genes in the two pyrimidine dimer excision repair pathways of Deinococcus radiodurans. $J$. Bacteriol. 156, 576-583.

Farr, S. B. and Kogoma, T. (1991) Oxidative stress responses in Escherichia coli and Salmonella typhimurium. Microbiol. Rev. 55, 561-585.

Fridovich, I. (1995) Superoxide radical and superoxide dismutase. Ann. Rev. Biochem. 64, 97-112.

Gersten, D. M. (1996) Gel Electrophoresis: Proteins, essential techniques, D. Rickwood (ed). Wiley \& Sons, West Sussex, UK.

Gregory, E. M., Yost Jr., F. J. and Fridovich, I. (1973) Superoxide dismutase of Escherichia coli intracellular localization and function. J. Bacteriol. 115, 987-991.

Gutman, P. D., Carroll, J. D., Masters, C. I. and Minton, K. W. (1994) Sequencing, targeted mutagenesis and expression of recA gene required for the extreme radioresistance of Deinococcus radiodurans. Gene 141, 31-37.

Halliwell, B. and Gutteridge, J. M. C. (1999) Free Radicals in Biology and Medicine (3rd ed.), pp. 105-350. Oxford Univ. Press, Oxford, UK.

Kim, E. -J., Kim, H. -P., Hah, Y. C. and Roe, J. -H. (1996) Differential expression of superoxide dismutases containing $\mathrm{Ni}$ and $\mathrm{Fe} / \mathrm{Zn}$ in Streptomyces coelicolor. Eur. J. Biochem. 241, 178-185.

Kim, J., Sharma, A. K., Abbott, S. N., Wood, E. A., Dwyer, D. W., Jambura, A., Minton, K. W., Inman, R. B., Daly, M. J. and Cox, M. M. (2002) RecA protein from the extremely radioresistant bacterium Deinococcus radiorurans: Expression, purification and characterization. J. Bacteriol. 184, 1649-1660.

Lee, I. J. and Lee, Y. N. (1995) Purification and characterization of catalase-3 of Deinococcus radiophilus ATCC 27603. J. Microbiol. 33, 239-243.

Li, T., Huang, X., Zhou, R., Liu, Y., Li, B., Nomura, C. and Zhao, J. (2002) Differential expression and localization of $\mathrm{Mn}$ and Fe superoxide dismutase in the heterocystous Cyanobacterium Anabaena sp. strain PCC 7120. J. Bacteriol. 184, 5096-5103.

Lowry, O. H., Rosebrough, N. J., Farr, A. C. and Randall, R. J. (1951) Protein measurement with the Folin phenol reagent. $J$. Biol. Chem. 193, 265-275.

Madigan, M. T., Martinko, J. M. and Parker, J. (2000) Brock Biology of Microorganisms. $9^{\text {th }}$ ed., P. 540. Prentice Hall, Upper Saddle River, NY, USA.

Makarova, K. S., Aravind, L., Wolf, Y. I., Tatusov, R. L., Minton, K. W., Koomin, E. V. and Daly, M. J. (2001) Genome of the extremely radiation resistant bacteria, Deinococcus radiodurans viewed from the perspective of comparative genomics. Microbiol. Mol. Biol. Rev. 65, 44-79.

Marklund, S. and Marklund, G. (1974) Assay of SOD by pyrogallol autotoxidation. Eur. J. Biochem. 47, 469-474.

Mueller, D. J., Baumeister, W. and Engel, W. A. (1996) Conformational change of the hexagonally packed intermediate layer of Deinococcus radiorurans monitored by atomic force microscopy. J. Bacteriol. 178, 3025-3030.

Murray, R. G. E. (1986). Family II, Deinococcaceae; in Bergeys Manual of Systematic Bacteriology, Buchanan, R. E. and Gibbons, N. E. (eds.), pp. 1035-43. The Williams and Wilkins Co., Baltimore, USA.

Narumi. I., Cherdchu, K., Kikuchi, S., Kitayama, T. and Watanabe, H. (1997) The Deinococcus radiodurans uvrA gene: 
identification of mutation sites of two mitomycin-sensitive strains and the first discovery of insertion sequence element from deinobacteria. Gene 198, 115-126.

Oh, K. A. and Lee, Y. N. (1998) Purification and characterization of catalase-2 of Deinococcus radiophilus ATCC 27603. J. Biochem. Mol. Biol. 31, 144-148.

Park, Y-B. and Han, Y-H. (1997) Increased activities of SOD and catalases on aerobic growth in Arcobacter nitrofigilis. $J$. Microbiol. 35, 239-240.

Schnell, S. and Steinman, H. M. (1995) Function and stationaryphase induction of periplasmic copper-zinc superoxide dismutase and catalase/peroxidase in Caulobacter crescentus. $J$. Bacteriol. 177, 5924-5929.

Soung, N. K. and Lee, Y. N. (2000) Iso-catalase profiles of Deinococcus spp. J. Biochem. Mol. Biol. 33, 412-416.

St. John, G. and Steinman, H. M. (1996) Periplasmic copper-zinc superoxide dismutase of Legionella pneumoniae: role in stationary phase survival. J. Bacteriol. 178, 1578-1584.
Storz, G. and Zheng, M. (2000) Oxidative stress; in Bacterial Stress Responses, Storz, G. and Hengge-Aronis, R. (eds.) pp. 47-59, ASM Press, Washington D.C., USA.

Tyrrell, R. M. (1991) UVA (320-380 nm) radiation as an oxidative stress; in Oxidative stress, oxidants and antioxidants, Sies, H. (ed.), pp. 57-83, Academic Press, London, UK.

White, O., Eisen, J. A., Heidelberg, J. F., Hickey, E. K. and 28 persons. (1999) Gemone sequence of the radioresistant bacterium Deinococcus radiodurans R1. Science 286, 15711577.

Youn, H. D., Kim, E. J., Hah, Y. C. and Kang, S. O. (1996) A novel nickel-containing superoxide dismutase from Streptomyces spp. Biochem. J. 318, 889-896.

Yun, E. J. and Lee, Y. N. (2000) Production of two different catalase-peroxidases by Deinococcus radiophilus. FEMS Microbiol. Lett. 184, 155-159.

Yun, Y. S. and Lee, Y. N. (2001) Superoxide dismutase profiles in the mesophilic Deinococcus species. J. Microbiol. 39, 232-235. 